\title{
THE PRESERVATION OF THE SEMIPRIME GOLDIE PROPERTY BY STRONG SEMILATTICE SUMS
}

\author{
YUE-CHAN P. HO
}

(Communicated by Warren J. Wong)

\begin{abstract}
Let $R$ be a strong semilattice sum of rings $R_{\alpha}(\alpha \in P)$ where $P$ is an m.u.-semilattice. When $P$ is infinite, $R$ is not a right Goldie ring; and when $P$ is finite, $R$ is semiprime right Goldie iff each $R_{\alpha}$ is semiprime right Goldie.
\end{abstract}

\section{INTRODUCTION}

Let $P$ be a semilattice whose natural order is indicated by <. As in [1], for any $\alpha, \beta$ in $P, \beta$ is said to be maximal under $\alpha$ if $\beta<\alpha$, and there is no other $\gamma$ in $P$ such that $\beta<\gamma<\alpha . P$ is called an m.u.-semilattice if the following conditions hold for $P$.

(1) If $\alpha, \gamma \in P$ with $\gamma<\alpha$, then there exists $\beta$ maximal under $\alpha$ such that $\gamma \leq \beta<\alpha$.

(2) The set $\{\beta \in P \mid \beta$ is maximal under $\alpha\}$ is finite for each $\alpha \in P$.

A ring $R$ is called a strong semilattice sum of rings $R_{\alpha}(\alpha \in P)$ by [2, p. 1018; 3 , p. 12] if there exists a family of homomorphisms $\varphi_{\alpha, \beta}: R_{\alpha} \rightarrow R_{\beta}(\alpha, \beta \in$ $P ; \alpha \geq \beta$ ) such that

(a) $R$ is the disjoint union of rings $R_{\alpha}(\alpha \in P)$;

(b) $\varphi_{\alpha, \alpha}$ is the identity map of $R_{\alpha}$;

(c) if $\alpha>\beta>\gamma$, then $\varphi_{\alpha, \beta} \varphi_{\beta, \gamma}=\varphi_{\alpha, \gamma}$;

(d) if $a \in R_{\alpha}$ and $b \in R_{\beta}$, then the multiplication in $R$ is determined by $a \cdot b=a \varphi_{\alpha, \alpha \beta} \cdot b \varphi_{\beta, \alpha \beta} \in R_{\alpha \beta}$.

M. L. Teply, E. G. Turman, and A. Quesada have shown that, when $P$ is an m.u.-semilattice, $R$ is $\pi$-semisimple iff each $R_{\alpha}$ is $\pi$-semisimple in [1]. We want to know if this is also true for the semiprime Goldie property.

\section{Preliminaries}

Throughout this paper we need the monomorphism $*: R_{\beta} \rightarrow R$ for each

Received by the editors August 16, 1990 and, in revised form, October 15, 1990; the contents of this paper have been presented by the author at the 97th Annual AMS-MAA meeting in San Francisco, January 18, 1991.

1980 Mathematics Subject Classification (1985 Revision). Primary 20M25; Secondary 16A12.

Key words and phrases. Semilattice sum, semiprime Goldie. 
$\beta \in P$ defined in [1] as

$$
\begin{aligned}
a^{*}= & a-\left[\sum_{|\Delta|=1} a \varphi_{\beta, \Delta^{\prime}}\right]+\sum_{|\Delta|=2} a \varphi_{\beta, \Delta^{\prime}} \\
& -\left[\sum_{|\Delta|=3} a \varphi_{\beta, \Delta^{\prime}}\right]+\cdots+(-1)^{n} a \varphi_{\beta, \beta_{1} \beta_{2} \cdots \beta_{n}},
\end{aligned}
$$

where $a \in R_{\beta}, \Delta$ is a nonempty subset of $\left\{\beta_{1}, \beta_{2}, \ldots, \beta_{n}\right\}$, which is the set of elements maximal under $\beta, \Delta^{\prime}$ denotes the product (g.l.b.) of all the $\beta_{i}$ in $\Delta$, and $|\Delta|$ is the cardinality of $\Delta$.

As shown in [1], if $\beta<\alpha$ or $\alpha$ and $\beta$ are not related by $<$, then for all $a \in R_{\alpha}$ and $b \in R_{\beta}, a^{*} b=0=b a^{*}$; and $a^{*} b=a^{*} b^{*}, b a^{*}=b^{*} a^{*}$ if $\alpha=\beta$. Also, when $A$ is a (resp. right or left) ideal of $R_{\alpha}, A^{*}$, which is isomorphic to $A$, is a (resp. right or left) ideal of $R$. Therefore, whenever we mention a property of $R_{\alpha}$ (resp. $a_{\alpha} \in R_{\alpha}$ ), $R_{\alpha}^{*}$ (resp. $a_{\alpha}^{*} \in R_{\alpha}^{*}$ ) has the same property, and vice versa.

Lemma 1. If $\alpha, \beta \in P$ and $\alpha \neq \beta$, then $a^{*} b^{*}=0=b^{*} a^{*}$, for all $a \in R_{\alpha}$ and $b \in R_{\beta}$.

Proof. $\alpha \neq \beta$ means either one of them is larger than the other, say $\alpha>\beta$, or $\alpha$ and $\beta$ are not related by $<$. Write $b^{*}=b+b_{1}+b_{2}+\cdots+b_{n}$, where $b_{i}=(-1)^{i} \sum_{|\Delta|=i} b \varphi_{\beta, \Delta^{\prime}}$. Then $a^{*} b=b a^{*}=a^{*} b_{i}=b_{i} a^{*}=0$ for all $i=$ $1, \ldots, n$ since either $\Delta^{\prime}<\alpha$ or $\Delta^{\prime}$ and $\alpha$ are not related by $<$. Therefore, $a^{*} b^{*}=b^{*} a^{*}=0$.

Lemma 2. If $T$ is a semiprime ring and there exists some $a \in T$ such that $a T=0$, then $a=0$.

Proof. Consider the right ideal $\langle a\rangle$ of $T$ generated by $a$. Since $\langle a\rangle=\langle\mathbb{Z} a+a T\rangle$ and $a^{2} \in a T=0$, we can get $(\langle a\rangle)^{2}=0$; i.e. $\langle a\rangle$ is nilpotent. But $T$ is semiprime, so $a \in\langle a\rangle=0$.

Lemma 3. If $T$ is a semiprime ring, $T^{\prime}$ is a two-sided ideal of $T$, and $I$ is an essential right ideal of $T$, then $I^{\prime}=I \cap T^{\prime}$ is an essential right ideal of $T^{\prime}$.

Proof. Suppose there is a nonzero right ideal $K$ of $T^{\prime}$ such that $K \cap I^{\prime}=0$. Then for all $0 \neq k \in K, k T$ is a right ideal of $T$. Since $T^{\prime}$ is an ideal of $T$, we have $(k T)^{2} \cap I=k T k T \cap I \subseteq k T^{\prime} \cap I \subseteq K \cap I^{\prime}=0$, which implies that $(k T)^{2}=0$; i.e. $k T$ is nilpotent. Hence, by Lemma $2, k=0$. But this is contradictory to the choice of $k$. Therefore $I^{\prime}$ is essential in $T^{\prime}$.

Lemma 4. If $R_{\alpha}$ is semiprime for each $\alpha \in P$ and $I_{\beta}^{*}$ is an essential right ideal of $R_{\beta}^{*}$, then the right ideal $I=I_{\beta}^{*}+\sum_{\alpha \neq \beta} R_{\alpha}^{*}$ is essential in $R$.

Proof. Suppose there is a nonzero right ideal $K$ of $R$ such that $K \cap I=0$. Then for all $0 \neq k \in K, k=\sum_{i=1}^{n} k_{\alpha_{i}}$ with $k_{\alpha_{i}} \in R_{\alpha_{i}} \backslash 0$ and $\alpha_{i} \in P$ for all $i=1, \ldots, n$. Now choose a maximal element $\alpha_{0}$ from the set $\left\{\alpha_{1}, \ldots, \alpha_{n}\right\}$. Then $k R_{\alpha_{0}}^{*}=k_{\alpha_{0}} R_{\alpha_{0}}^{*}=k_{\alpha_{0}}^{*} R_{\alpha_{0}}^{*}$. If $\alpha_{0}=\beta$, then

$$
k_{\alpha_{0}}^{*} R_{\alpha_{0}}^{*} \cap I_{\beta} \subseteq K \cap I=0 ;
$$

and when $\alpha_{0} \neq \beta, k_{\alpha_{0}}^{*} R_{\alpha_{0}}^{*} \cap R_{\alpha_{0}}^{*}=k R_{\alpha_{0}}^{*} \cap R_{\alpha_{0}}^{*} \subseteq K \cap I=0$ because $R_{\alpha_{0}}^{*} \subseteq I=$ $I_{\beta}^{*}+\sum_{\alpha \neq \beta} R_{\alpha}^{*}$. Therefore $k_{\alpha_{0}}^{*} R_{\alpha_{0}}^{*}=0$ in both cases. And so $k_{\alpha_{0}}^{*}=0$ by Lemma 
2; i.e. $k_{\alpha_{0}}=0$. But this contradicts the assumption that $k_{\alpha_{0}} \neq 0$. Hence $I$ must be essential in $R$.

If $P$ is a finite semilattice, we can always find minimal elements in $P$. Furthermore, there is only one minimal element in $P$, for if both $\alpha$ and $\beta$ are minimal elements then $\alpha \beta \leq \alpha, \beta$ implies that $\alpha \beta=\alpha=\beta$. Thus we denote the unique minimal element in $P$ by 0 . Also, by the definition of $*$, we have $R_{0}=R_{0}^{*}$ and $a_{0}=a_{0}^{*}$ for all $a_{0} \in R_{0}$.

The following lemmas are proved to be true when $P$ is finite.

Lemma 5. $R=\bigoplus_{\alpha \in P} R_{\alpha}^{*}$.

This lemma is a special case of Theorem 3.7 in [3]. However, since the proof is not readily accessible, we still show our work here.

Proof. First, we have $\sum_{\alpha \in P} R_{\alpha}^{*} \subseteq R$ because each $R_{\alpha}^{*}$ is an ideal of $R$. Next, for any $a \in R, a=\sum_{\alpha \in P} a_{\alpha}$ with $a_{\alpha} \in R_{\alpha}$ for each $\alpha$. Let $M_{1}$ be the set of maximal elements in $P$. Then for each $\beta \in M_{1}, a_{\beta}^{*}=a_{\beta}+a_{1}+\cdots+a_{t}$ with $a_{\beta} \in R_{\beta}$ and $a_{i}=(-1)^{i} \sum_{|\Delta|=i} a_{\beta} \varphi_{\beta, \Delta^{\prime}}$ implies that

$$
a_{\beta}=a_{\beta}^{*}-a_{1}-\cdots-a_{t} \text {. }
$$

Hence $a=\sum_{\beta \in M_{1}} a_{\beta}^{*}+\sum_{\alpha \in P_{1}} b_{\alpha}$ with $b_{\alpha} \in R_{\alpha}$ and $P_{1}=P \backslash M_{1}$. Therefore $a \in \sum_{\alpha \in P} R_{\alpha}^{*}$ iff $\sum_{\alpha \in P_{1}} b_{\alpha} \in \sum_{\alpha \in P} R_{\alpha}^{*}$. Since $P$ is finite and $R_{0}=R_{0}^{*}$, we can repeat the procedure for $\sum_{\alpha \in P_{1}} b_{\alpha}$ and finally, have $a \in \sum_{\alpha \in P} R_{\alpha}^{*}$. So by Lemma 1 and the fact that $R_{\alpha}^{*} \cap R_{\beta}^{*}=0$ whenever $\alpha \neq \beta$, we have $R=\bigoplus_{\alpha \in P} R_{\alpha}^{*}$.

Lemma 6. $a$ is regular in $R$ iff $a=\sum_{\alpha \in P} a_{\alpha}^{*}$ where $a_{\alpha}^{*}$ is regular in $R_{\alpha}^{*}$ for each $\alpha$ in $P$.

Proof. $a \in R$ implies that $a=\sum_{\alpha \in P} a_{\alpha}^{*}$ with $a_{\alpha}^{*} \in R_{\alpha}^{*}$ for each $\alpha$ in $P$ by Lemma 5. Suppose there is $\beta$ in $P$ such that $a_{\beta}^{*}$ is not regular in $R_{\beta}^{*}$; i.e. there exists $0 \neq b_{\beta}^{*} \in R_{\beta}^{*}$ such that $a_{\beta}^{*} \cdot b_{\beta}^{*}=0$ (resp. $b_{\beta}^{*} \cdot a_{\beta}^{*}=0$ ). Then $a \cdot b_{\beta}^{*}=a_{\beta}^{*} \cdot b_{\beta}^{*}=0$ (resp. $b_{\beta}^{*} \cdot a=0$ ) contradicts that $a$ is regular in $R$.

On the other hand, if each $a_{\alpha}^{*}$ is regular in $R_{\alpha}^{*}$ and if there is $0 \neq b \in R$ such that $a b=0$ (resp. $b a=0$ ) where $a=\sum_{\alpha \in P} a_{\alpha}^{*}$, write $b=\sum_{\alpha \in P} b_{\alpha}^{*}$, then

$$
0=a b=\left[\sum_{\alpha \in P} a_{\alpha}^{*}\right] \cdot\left[\sum_{\alpha \in P} b_{\alpha}^{*}\right]=\sum_{\alpha \in P}\left(a_{\alpha}^{*} \cdot b_{\alpha}^{*}\right)
$$

implies $a_{\alpha}^{*} \cdot b_{\alpha}^{*}=0$ (resp. $b_{\alpha}^{*} \cdot a_{\alpha}^{*}=0$ ) for each $\alpha$; i.e. every $b_{\alpha}^{*}=0$, which is a contradiction.

\section{MAin Results}

Theorem 1. $R$ is not a right Goldie ring when $P$ is infinite.

Proof. Let $M=\left\{\alpha_{1}, \alpha_{2}, \alpha_{3}, \ldots\right\}$ be an infinite subset of $P$, and let $I_{n}=$ $\sum_{i=1}^{n} R_{\alpha_{i}}^{*}$ for each positive integer $n$. Since each $R_{\alpha_{i}}^{*}$ is an ideal of $R$, so is each $I_{n}$. Then, by Lemma $1, I_{1} \varsubsetneqq I_{2} \varsubsetneqq I_{3} \varsubsetneqq \cdots$ is an infinite ascending chain of annihilators in $R$. Hence $R$ is not a right Goldie ring.

To show a ring is semiprime right Goldie, we are going to apply Goldie's Theorem [4, Chapter 7]. 
Theorem 2. If $P$ is finite, $R$ is semiprime right Goldie iff each $R_{\alpha}$ is semiprime right Goldie.

Proof. Assume $R$ is semiprime right Goldie. For each $\beta \in P$, let $I_{\beta}^{*}$ be any essential right ideal of $R_{\beta}^{*}$, and let $I=I_{\beta}^{*}+\sum_{\alpha \neq \beta} R_{\alpha}^{*}$. Then $I$ is an essential right ideal of $R$ by Lemma 4. Also, $I$ contains a regular element $a$ of $R$ by Goldie's Theorem. By Lemma 6, we can write $a=\sum_{\alpha \in P} a_{\alpha}^{*}$ where each $a_{\alpha}^{*}$ is regular in $R_{\alpha}^{*}$. Then $a_{\beta}^{*} \in I_{\beta}^{*}$ since both $a$ and $\sum_{\alpha \neq \beta} a_{\alpha}^{*}$ are in $I$.

Since $R$ itself is an essential right ideal, $R$ has a regular element by Goldie's Theorem. So each $R_{\alpha}^{*}$ contains a regular element by Lemma 6 . Let $a_{\beta}^{*}$ be a regular element in $R_{\beta}^{*}$, choose any regular element $a_{\alpha}^{*}$ from $R_{\alpha}^{*}$ for each $\alpha \neq \beta$, and let $a=\sum_{\alpha \in P} a_{\alpha}^{*}$. Then $a$ is regular in $R$. Hence $a R$ is an essential right ideal of $R$ by [4, Lemma 7.2.3]. Also, $a_{\beta}^{*} R_{\beta}^{*}=\left[\sum_{\alpha \in P} a_{\alpha}^{*} R_{\alpha}^{*}\right] \cap R_{\beta}^{*}=$ $a R \cap R_{\beta}^{*}$ shows that $a_{\beta}^{*} R_{\beta}^{*}$ is an essential right ideal of $R_{\beta}^{*}$ by Lemma 3 . Whence $R_{\beta}^{*}$ is, and so $R_{\beta}$ is, semiprime right Goldie.

Conversely, suppose each $R_{\alpha}$ is semiprime right Goldie, and $I$ is any essential right ideal of $R$. For each $\alpha$, let $I_{\alpha}^{*}=I \cap R_{\alpha}^{*} . I_{\alpha}^{*} \neq 0$ because $I$ is essential and $R_{\alpha}^{*}$ is a nonzero ideal in $R$. So $I_{\alpha}^{*}$ is an essential right ideal in $R_{\alpha}^{*}$ by Lemma 3. Then $I_{\alpha}^{*}$ contains a regular element $a_{\alpha}^{*}$ of $R_{\alpha}^{*}$. Therefore $a=\sum_{\alpha \in P} a_{\alpha}^{*}$ is a regular element in $R$ and is contained in $I$.

For each regular element $a$ in $R, a=\sum_{\alpha \in P} a_{\alpha}^{*}$ with $a_{\alpha}^{*}$ regular in $R_{\alpha}^{*}$ for each $\alpha$. Suppose there is a nonzero right ideal $K$ of $R$ such that $K \cap a R=0$. Then for any nonzero $k \in K$, with $k=\sum_{\alpha \in P} k_{\alpha}^{*}, k_{\beta}^{*} R_{\beta}^{*} \cap a_{\beta}^{*} R_{\beta}^{*} \subseteq K \cap a R=0$ implies $k_{\beta}^{*}=0$ by Lemma 2 for each $\beta$, which contradicts the choice of a nonzero $k$. Hence $a R$ is an essential right ideal of $R$. Therefore $R$ is semiprime right Goldie.

\section{ACKNOWLEDGMENT}

The author would like to express her appreciation to Robert H. Oehmke for his assistance with this problem.

\section{REFERENCES}

1. M. L. Teply, E. G. Turman, and A. Quesada, On semisimple semigroup rings, Proc. Amer. Math. Soc. 79 (1980), 157-163.

2. H. L. Chick and B. J. Gardner, The preservation of some ring properties by semilattice sums, Comm. Algebra 15 (1987), 1017-1038.

3. E. G. Turman, Supplementary semilattice sums of rings, Ph.D. dissertation, Univ. of Florida, 1980.

4. I. N. Herstein, Noncommutative rings, Math. Assoc. Amer., distributed by J. Wiley, 1968.

5. T. W. Hungerford, Algebra, Springer-Verlag, New York, 1974.

6. B. Stenström, Rings of quotients, Springer-Verlag, New York, 1975.

Department of Mathematics, The University of Iowa, Iowa City, Iowa 52242 